ANNALS OF “DUNAREA DE JOS” UNIVERSITY OF GALATI
MATHEMATICS, PHYSICS, THEORETICAL MECHANICS
FASCICLE II, YEAR XIII (XLIV) 2021, No. 1
DOI: https://doi.org/10.35219/ann-ugal-math-phys-mec.2021.1.05

\title{
Comparative study of DFT, AM1, and PM3 optimization methods modeling new psychotropic amphetamines
}

\author{
Adelina Ion ${ }^{1, *}$, Mirela Praisler ${ }^{1,2}$, Steluta Gosav ${ }^{1,2}$ \\ I "Dunarea de Jos" University of Galati, Faculty of Sciences and Environment, 47 Domneasca Street, 800008, \\ Galati, Romania, \\ 2 "Dunarea de Jos" University of Galati, Faculty of Sciences and Environment, INPOLDE research center, \\ 800008 Galati, Romania \\ *Corresponding author: Adelina.Ion@ugal.ro
}

\begin{abstract}
In order to improve the determination of physico-chemical descriptors of organic substances, it is necessary to optimize the computational representations of their molecular structures. The computerized estimation of these descriptors is especially useful in the case of drugs of abuse, as clinical studies are not recommended for new compounds belonging to classes known to have a high toxicity. The optimization of molecular structures allows the improvement of the accuracy in determining the physico-chemical descriptors and consequently of the associated quantitative structure - property or structure - activity relationships (QSAR). We are presenting a comparative study regarding the effect of the AM1, PM3, and DFT optimization methods, which were applied in the case of a series of new psychotropic amphetamines for their physico-chemical characterization based on molecular descriptors.
\end{abstract}

Keywords: psychotropic amphetamines, molecular structure optimization.

\section{INTRODUCTION}

The description of the molecular structure of large molecules, as well as their properties, has always been a problem, due to the fact that the methods of quantum mechanics could not be used as long as the capacity of the computing hardware was not developed enough. With the development of molecular mechanics and high-speed computing capabilities, the use of techniques involving molecular energy assessments (e. g. optimization of the molecular geometry, molecular dynamics, or conformational study) has become accessible.

For the calculation of molecular properties, a correctly determined molecular structure is required and for the fundamental state, a geometry corresponding to the minimum energy. Thus, the determination of an optimized geometry of a chemical compound leads to a new stable structure, which is the basis for quantum calculations from which large sets of structural and electronic properties may be determined. At the same time, a rigorous initial structure is required in the application of optimization procedures. In addition, an optimized molecular structure allows a more efficient determination of physico-chemical descriptors and, therefore, of more precise quantitative structure-activity relationships (QSAR).

Because semi-empirical methods AM1 (Austin Method 1) and PM3 (Parametrization Method 3) may provide standard information about the analyzed molecules, they represent a perfect combination of ab initio methods and data from experimental studies [1-2]. The DFT approach is currently a powerful and extremely credible tool for determining the various molecular properties. In particular, the B3LYP functional hybrid provides high accuracy and computational efficiency for the vibrational spectra of large and medium-sized molecules [3]. DFT can also be defined as a computational method 
of quantum mechanical modeling, which has applications, especially in physics, chemistry, and materials science. This method has been successfully applied for the investigation of the electronic structure of molecular systems that have several atoms, i.e. in the research of the fundamental states of a molecular system [4].

This paper presents an experimental study in which the optimization methods AM1, PM3, and DFT have been applied to a series of seven new hallucinogenic drugs of abuse in order to perform their physico-chemical characterization based on molecular descriptors.

\section{EXPERIMENTAL}

The hallucinogenic amphetamines analyzed in this study are: 3,4methylenedioxymethamphetamine (C1); 7-(2-aminopropyl)benzofuran (C2); 5,6-methylenedioxy-2aminoindane (C3); 3,4-Methylenedioxypyrovalerone (C4); 1-(benzofuran-5-yl)-N-methylpropan-2amine) (C5); N-Ethyl-4-methoxyamphetamine (C6); N-ethylpentylone (C7). The molecules of these seven compounds were represented in 3D coordinates, and their geometries were completely optimized by using the semi-empirical methods AM1 and PM3, as well as the quantum method DFT.

The programs used for the optimization process are: HyperChem 8.03 [5], which was used to apply the Austin Model 1 (AM1) and Parametrization Model 3 (PM3) methods, and Gaussian 09 [6], which was used for the application of the density functional theory (DFT) method.

\section{RESULTS AND DISCUSSION}

Figure 1 presents the optimized molecular structure of N-ethylpentilone (C7), which was obtained by using the Molden 5.0 program [7] following the optimization performed by applying the DFT method (B3LYP hybrid functional and the basic set 6-311G $(\mathrm{d}, \mathrm{p})$ ).

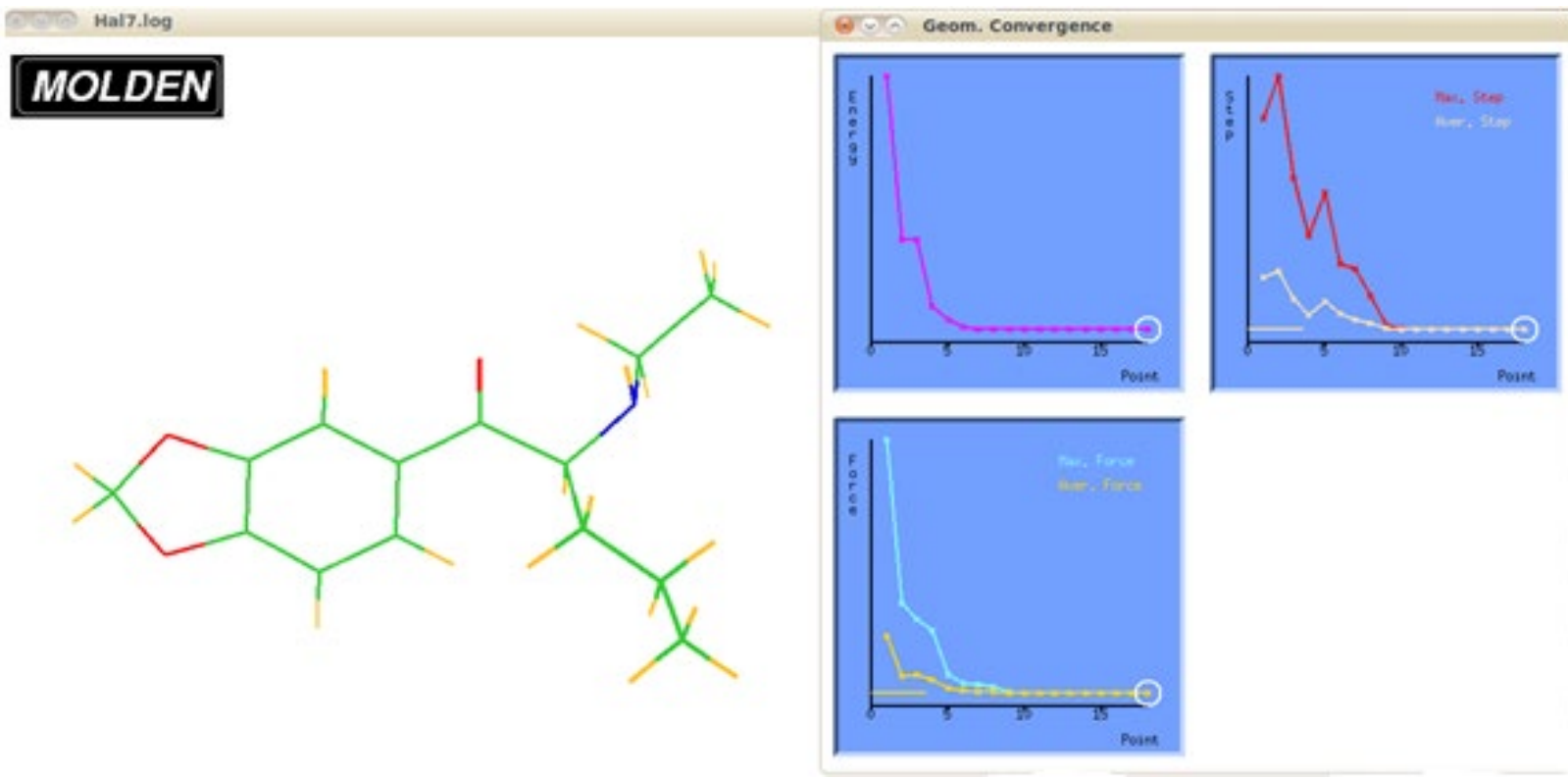

Fig. 1. Optimized molecular structure of N-ethylpentylone (C7), obtained by using the Molden 5.0 program following the optimization performed by applying the DFT method (functional hybrid B3LYP and basic set 6-311G $(d, p))[8]$.

The Gibbs free energy obtained as a result of the optimization of the molecular structures of the studied hallucinogenic amphetamines by applying the AM1, PM3 and DFT / B3LYP / 6-311G (d, p) methods are presented in Table 1. The latter shows that the Gibbs free energy values obtained by applying the semi-empirical method AM1 are lower than those obtained with the PM3 method. This 
indicates that, in the case of the studied compounds, AM1 is more appropriate for the semi-empirical optimization of the molecular structures.

The DFT quantum method (B3LYP / 6-311G $(\mathrm{d}, \mathrm{p})$ ) is widely used, as it is a precise way to optimize molecular structures, to calculate energies, frequencies, and different molecular properties, at very low costs. The functional hybrid B3LYP method, in combination with the basic set $6-311 \mathrm{G}(\mathrm{d}, \mathrm{p})$, is very useful for simulating the interactions via $\mathrm{H}$ bonds in self-assembly systems. Indeed, Table 1 indicates that the DFT / B3LYP / 6-311G (d, p) method provides the lowest Gibbs free energy results in the case of the investigated hallucinogenic amphetamines as well.

Table 1. Gibbs free energy calculated by using the AM1, PM3 and DFT / B3LYP / 6-311G (d, p) optimization methods [8].

\begin{tabular}{|c|c|c|c|c|}
\hline Code & $\begin{array}{l}\text { Name and optimized molecular } \\
\text { structure of the controlled substance }\end{array}$ & $\begin{array}{c}\begin{array}{c}\text { Gibbs } \\
\text { free energy }\end{array} \\
\text { AM1 } \\
\text { (Hartree) } \\
\end{array}$ & $\begin{array}{c}\text { Gibbs } \\
\text { free energy } \\
\text { PM3 } \\
\text { (Hartree) } \\
\end{array}$ & $\begin{array}{c}\text { Gibbs } \\
\text { free energy } \\
\text { DFT/B3LYP/ } \\
\text { 6- 311G (d, p) } \\
\text { (Hartree) }\end{array}$ \\
\hline C1 & 3,4-methylenedioxymethamphetamine & -84.77 & -78.44 & -594.04 \\
\hline $\mathrm{C2}$ & 7-(2-aminopropyl)benzofuran & -77.67 & -71.98 & -556.92 \\
\hline $\mathbf{C 3}$ & 5,6-methylenedioxy-2-aminoindane & -83.75 & -77.30 & -592.85 \\
\hline C4 & 3,4-Methylenedioxypy & -128.81 & -119.90 & -902.67 \\
\hline $\mathrm{C5}$ & $\begin{array}{l}\text { 1-(benzofuran-5-yl)- } \mathrm{N} \text {-methylpropan-2- } \\
\text { amine) }\end{array}$ & -83.38 & -77.48 & -596.21 \\
\hline C6 & N-Ethyl-4-methoxyamphetamine & -85.46 & -79.78 & -598.59 \\
\hline
\end{tabular}




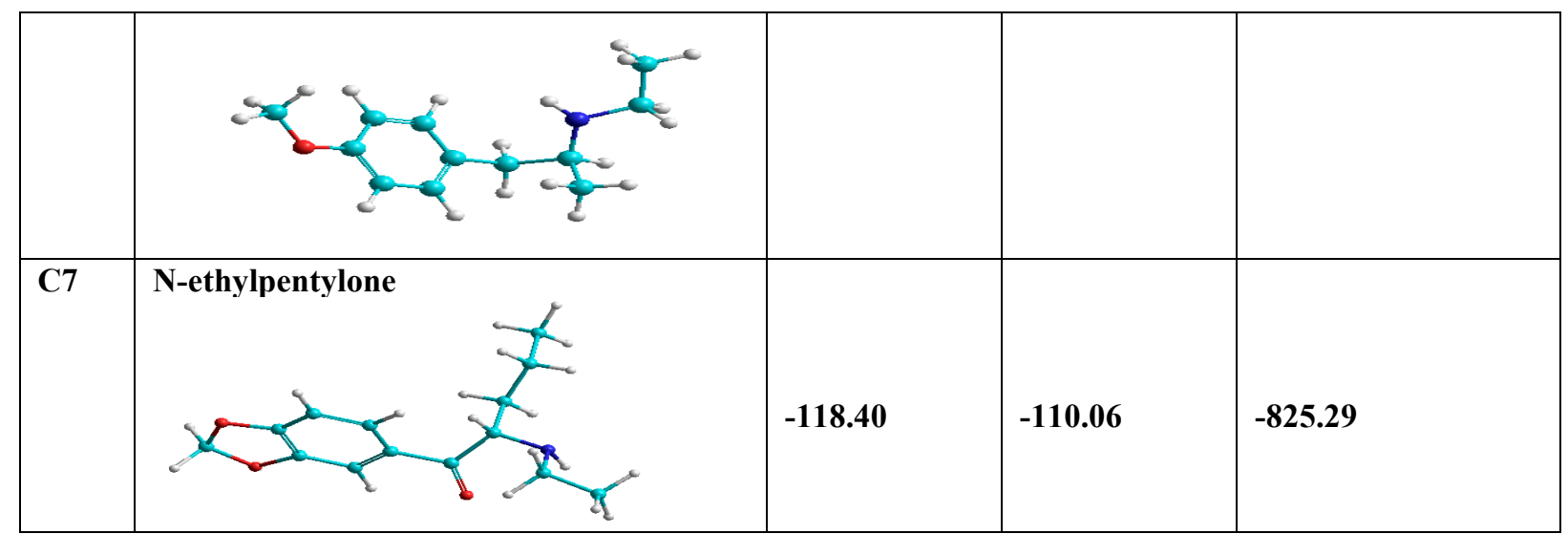

\section{CONCLUSIONS}

The structures optimized by using the DFT method allow a more accurate determination of their physico-chemical descriptors and QSAR. This approach is particularly important in the analysis of substances of abuse, such as the studied hallucinogenic amphetamines, as these compounds are extremely toxic and therefore clinical trials are not recommended.

The analysis of the Gibbs free energy values has indicated that, in the case of the studied drugs of abuse, the results obtained with the AM1 method for the optimization of these molecular structures are better than those obtained with the PM3 method. Nevertheless, the DFT / B3LYP / 6-311G (d, p) method led to the lowest Gibbs free energy results, and hence is the most appropriate method to be used in the process of optimizing the molecular structures of the investigated hallucinogenic amphetamines.

\section{References}

1. Christensen A. S., Kubař T., Cui Q., Elstner M., Semiempirical Quantum Mechanical Methods for Noncovalent Interactions for Chemical and Biochemical Applications, Chemical Reviews 116 (2016) 5301-5337.

2. http://www.scritub.com/stiinta/chimie/MECANICA-MOLECULARA24836.php

3. Korth H.G., de Heer M.I., Mulder P., A DFT study on intramolecular hydrogen bonding in 2substituted phenols: conformations, enthalpies, and correlation with solute parameters, Journal of Physical Chemistry A 106 (2002) 8779-8789.

4. Reveles J.U., Köster A.M., Geometry optimization in density functional methods, Journal of Computational Chemistry 25 (2004) 1109-1116.

5. Hyperchem software, Version 8.0.3., Hyper Co., USA, 2007.

6. Gaussian 09 software package, Revision D.01, Frisch M. J., et al., Gaussian Inc., Wallingford CT, 2009.

7. Schaftenaar G., Noordik, Molden J.H., A pre- and post-processing program for molecular and electronic structures, Journal of Computer-Aided Molecular Design 14 (2000) 123-134.

8. https://www.ugal.ro/files/doctorat/sustineri/2020/AdelinaION_rezumatPHD_030820.pdf 